\title{
Simulation of a Mobile Manipulator on Webots
}

\author{
https://doi.org/10.3991/ijoe.v14i02.7789 \\ Oscar F Avilés $\mathrm{S}^{\bowtie}$, Oscar G Rubiano M, Mauricio F Mauledoux M, \\ Angie J Valencia C, Robinson Jiménez Moreno \\ Militar Nueva Granada University, Bogotá, Colombia \\ oscar.aviles@unimilitar.edu.co
}

\begin{abstract}
A mobile manipulator is a mobile platform with a mounted serial manipulator. A Mobile Manipulator is a system subject to its kinematic restrictions and the degrees of freedom of the manipulator arm mounted on it. These systems combine the advantages of mobile platforms and robotic arms, and reduce their disadvantages. For example, a mobile manipulator has larger working space when it has a mobile platform, as it offers more functionality during operation [1], [2]. For the previously mentioned in this work will be shown the implementation in the robotic simulation software Webots, a mobile manipulator that allows to determine its operation in a virtual environment.
\end{abstract}

Keywords-Direct Kinematics, Inverse kinematics, Webots, Mobile manipulator

\section{$1 \quad$ Introduction}

Nowadays, movement planning and control of these systems have attracted a lot of attention due to their usefulness. The control of the mobile manipulators mainly includes two aspects: movement planning, both for the mobile platform and the manipulator, and the other, coordinated control of both. Once you have these two, the next step is to assign the task. In many cases mobilizing the manipulator results in kinematic redundancy, this can be solved by coordinating the platform and the manipulator. The type of coordination to be used will depend on the task specifications.

Some of the most important applications of mobile manipulators are: performing tasks involving explosives, hazardous environments such as outdoor exploration, space operations, construction, military tasks and personal service. The applications of robotic systems in hazardous environments have a high spectrum and their solutions are equally varied. In general, many applications include specific challenges associated with the uncertainty and nature of the tasks to be performed. Between different tasks there is the task of demining, which consists of eradicating anti-personnel mines or handling explosives, this task has a high humanitarian impact. On the other side, there is the handling of hazardous materials such as radioactive materials, corrosive or highly polluting materials, for which there is a risk if an operator performs these tasks [3]. 
For this reason, it is necessary to validate in some way the operation of the mechanisms and/or mathematical algorithms designed for this type of system, so Webots which is a robotic simulation system is appropriate for this type of testing.

\section{Description of the mobile manipulator}

The mobile platform has a Rocker - Bogie type suspension which has six independent traction wheels of which four are directionable, this is grouped in two trains, each one of which consists of three wheels as shown in Figure 1. To analyze it in a simpler way, two parts can be divided: on the mobile platform and the manipulator, this can be done by taking the origin of the manipulator on the mobile platform.

Each of these trains is supported by an articulated structure. The rear wheels are attached to the robot's body by means of a rigid arm known as Rocker and Rocker is attached by means of a pivot to an arm known as Bogie, which holds the middle and front wheels [4], [5]. The related parts can be seen in detail in Figure 2.

In addition, it has a differential system that is joined by means of arms connected to the Rocker and the structure which allows the platform to be always balanced even though the trains are at different heights [6].

On the other hand, the manipulator is a mechanism consisting of a sequence of rigid bodies called links. The physical structure of most robots is similar to the anatomy of the human arm, and it sometimes refers to different elements such as the arm, elbow and wrist [7]. The manipulator is five degrees of freedom as shown in Figure 3.

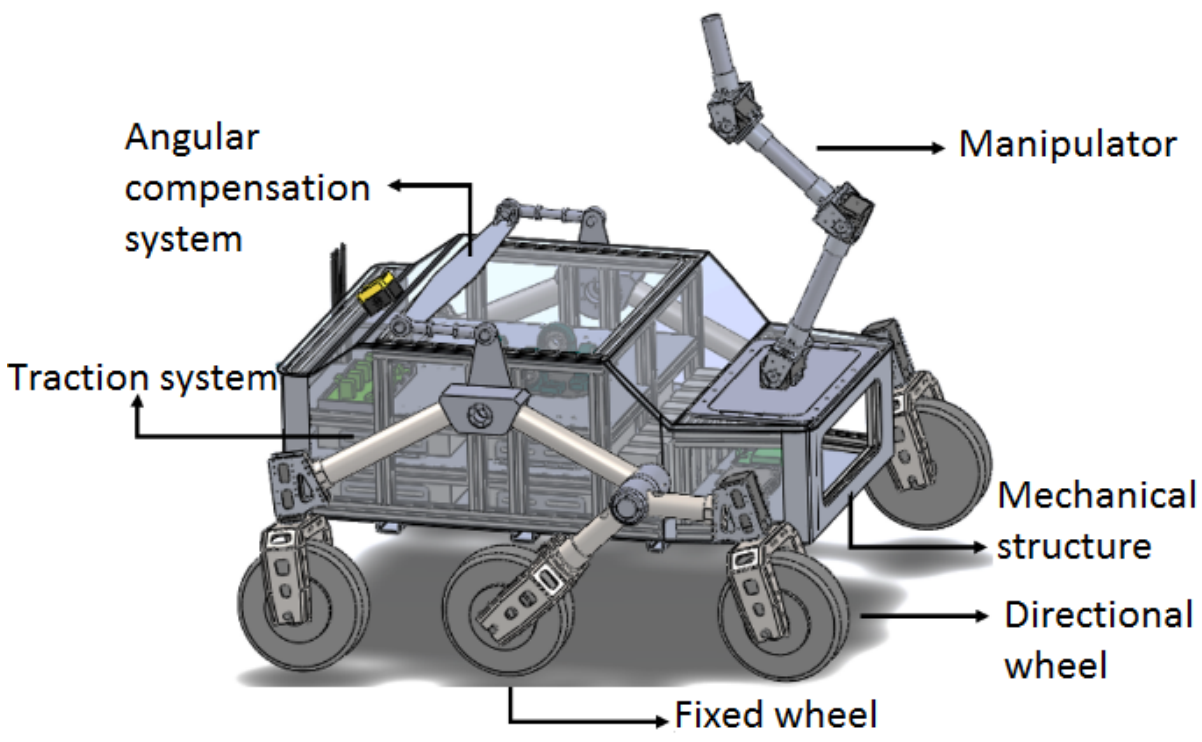

Fig. 1. CAD design of the mobile manipulator. 


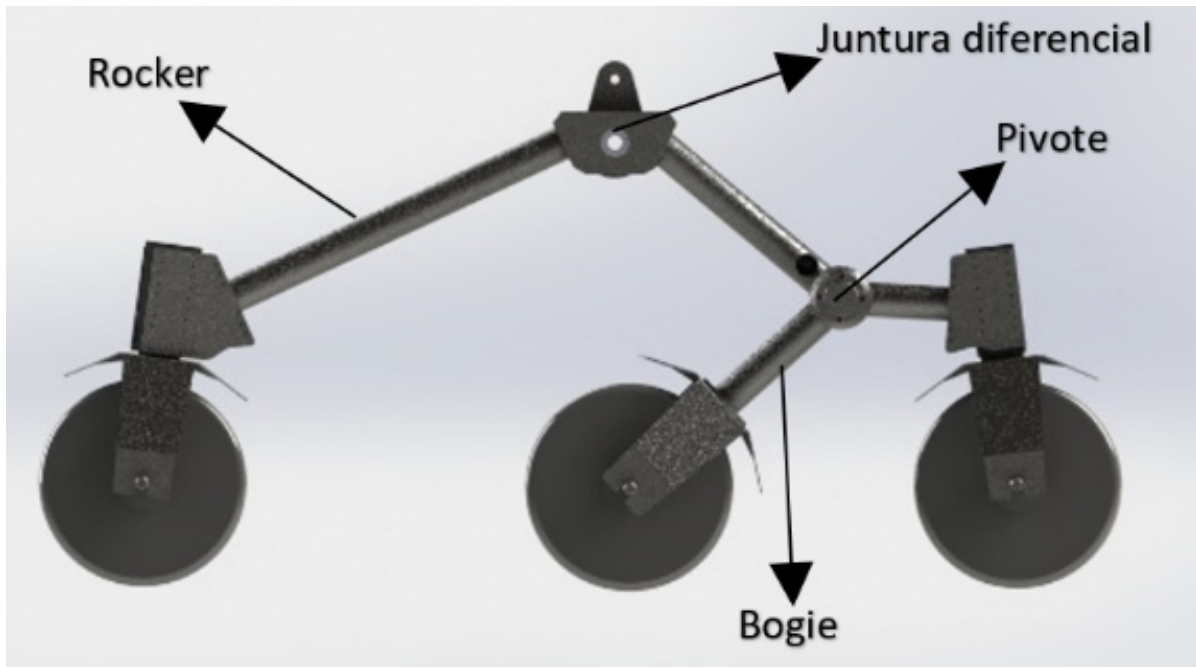

Fig. 2. Rocker -Bogie suspension.

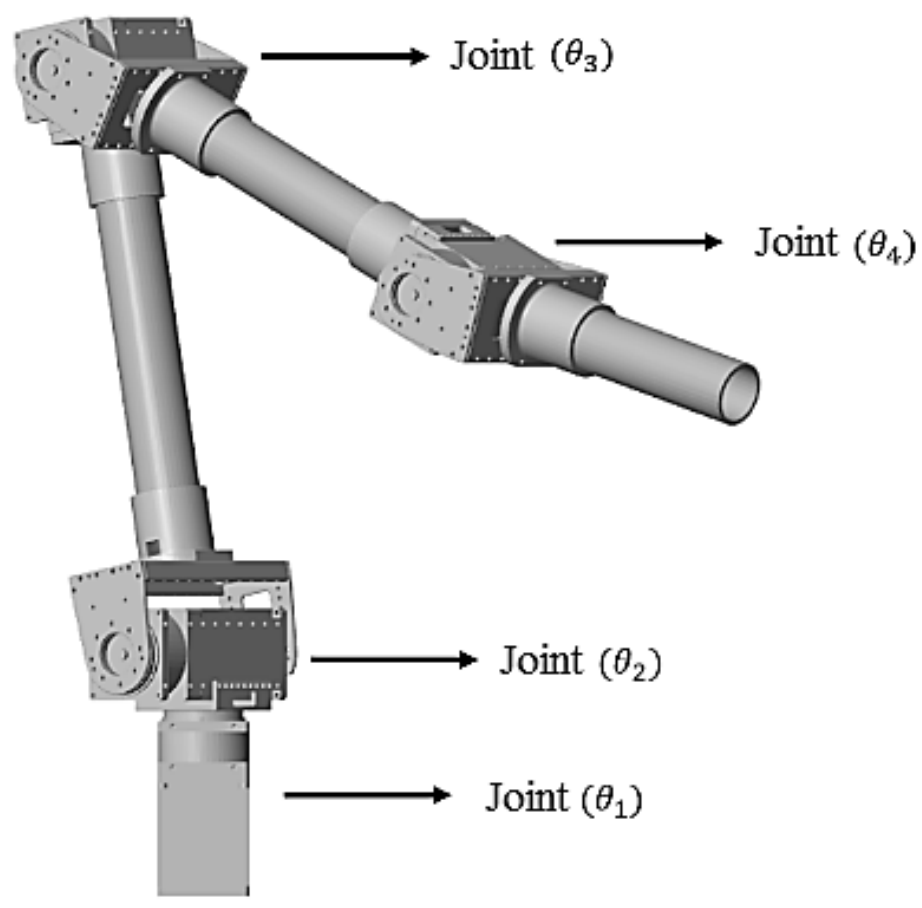

Fig. 3. Manipulator of 5 degrees freedom. 


\section{Robot Kinematics Manipulator}

The direct kinematics allows to determine the final position and orientation of the manipulator's final effector, knowing the value of each joint and its geometric parameters. For this purpose, the use of Denavit-Hartenberg is proposed, which makes it possible to establish the relative transformations between links. Table 1 shows the Denavit-Hartenberg parameter matrix for the robot to be simulated.

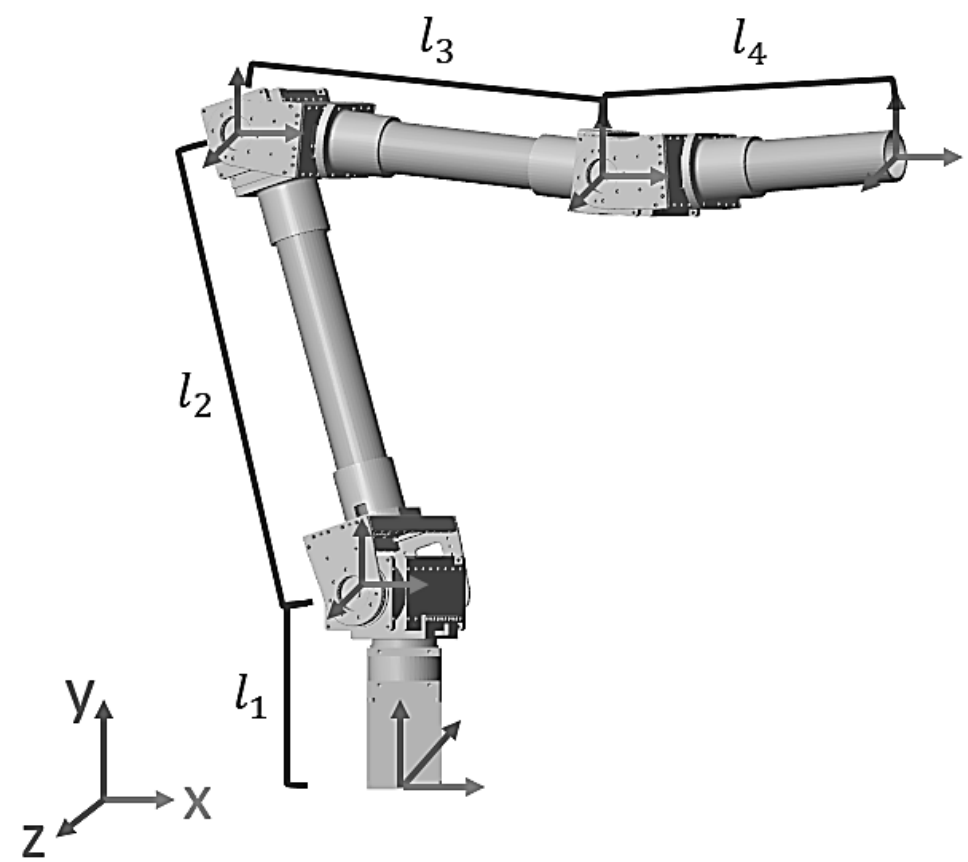

Fig. 4. Diagram of a manipulator with 5 degrees of freedom

Table 1. Denavit-Hartenberg parameter matrix.

\begin{tabular}{rcccc}
\hline Link & $\boldsymbol{\theta}_{\boldsymbol{i}}$ & $\boldsymbol{d}_{\boldsymbol{i}}$ & $\boldsymbol{a}_{\boldsymbol{i}}$ & $\boldsymbol{\alpha}_{\boldsymbol{i}}$ \\
\hline \hline 1 & $\theta_{1}$ & $l_{1}$ & 0 & 90 \\
\hline 2 & $\theta_{2}$ & 0 & $l_{2}$ & 0 \\
\hline 3 & $\theta_{3}$ & 0 & $l_{3}$ & 0 \\
\hline 4 & $\theta_{4}$ & 0 & $l_{4}$ & -90 \\
\hline 5 & $\theta_{5}$ & $l_{5}$ & 0 & 0 \\
\hline
\end{tabular}

From this matrix, the transformation matrices are proposed that will allow to relate the coordinate systems from one degree of freedom to the next. Eq. (1), Eq. (2), Eq. (3), Eq. (4), Eq. (5) show transformation matrixes of the coordinate system 0 to 1,1 to 2,2 to 3,3 to 4 and 4 to 5 . 


$$
\begin{gathered}
T_{01}=\left[\begin{array}{cccc}
c_{1} & 0 & s_{1} & 0 \\
s_{1} & 0 & -c_{1} & 0 \\
0 & 1 & 0 & l_{1} \\
0 & 0 & 0 & 1
\end{array}\right] \\
T_{12}=\left[\begin{array}{cccc}
c_{2} & -s_{2} & 0 & l_{2} \cdot c_{2} \\
s_{2} & c_{2} & 0 & l_{2} \cdot s_{2} \\
0 & 0 & 1 & 0 \\
0 & 0 & 0 & 1
\end{array}\right] \\
T_{23}=\left[\begin{array}{cccc}
c_{3} & -s_{3} & 0 & l_{3} \cdot c_{3} \\
s_{3} & c_{3} & 0 & l_{3} \cdot s_{3} \\
0 & 0 & 1 & 0 \\
0 & 0 & 0 & 1
\end{array}\right] \\
T_{23}=\left[\begin{array}{cccc}
0 & -s_{4} & c_{4} & l_{4} \cdot c_{4} \\
0 & c_{4} & s_{4} & l_{4} \cdot s_{4} \\
-1 & 0 & 0 & 0 \\
0 & 0 & 0 & 1
\end{array}\right] \\
T_{45}=\left[\begin{array}{cccc}
c_{5} & -s_{5} & 0 & 0 \\
s_{5} & c_{5} & 0 & 0 \\
0 & 0 & 1 & l_{5} \\
0 & 0 & 0 & 1
\end{array}\right]
\end{gathered}
$$

From this, the pre-multiplication of these matrices is carried out, which makes it possible to find the transformation matrix $T_{05}$, which describes the system kinematics with respect to the reference coordinate system. From this matrix, vector $P$ is extracted since it contains the position of the final link of the manipulator in $X, Y, Z$.

$$
T_{05}=\left[\begin{array}{cc}
R_{3 \times 3} & P_{3 \times 3} \\
F_{1 \times 3} & W_{1 \times 1}
\end{array}\right]=\left[\begin{array}{cc}
\text { Rot } & \text { Tras } \\
\text { Persp } & F_{\text {escala }}
\end{array}\right]
$$

\section{$4 \quad$ Mobile Robot Kinematics}

For the analysis of the mobile platform kinematics, the configuration scheme is defined to obtain the robot's kinematic model in which the movement restrictions of the independent wheels are expressed, by means of the physical decomposition of the robotic platform in order to define the wheels and their configurations. Thus, in Figure 5 Six-wheel robotic platform, the configuration to be handled is shown and Table 2 defines the variables that characterize it. 


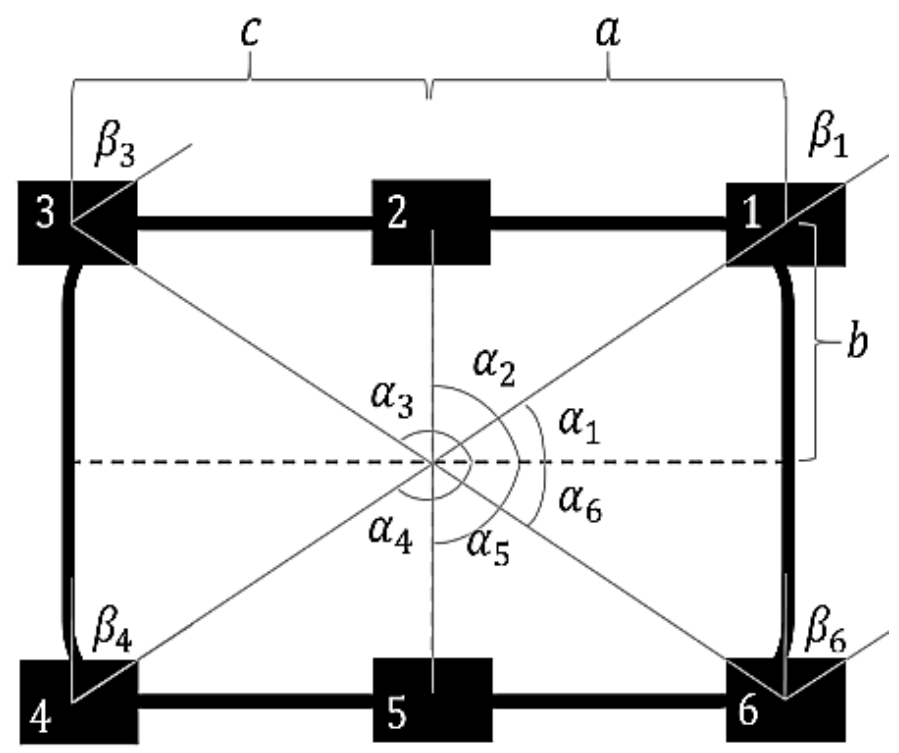

Fig. 5. Six-wheel robotic platform

Table 2. Angular Specification Matrix

\begin{tabular}{cccc}
\hline Ang & Value & Ang & Value \\
\hline$\propto_{1}$ & $\tan ^{-1}\left(\frac{b}{2 a}\right)$ & $\beta_{1}$ & $\left(\frac{\pi}{2}+\gamma_{1}\right)-\propto_{1}$ \\
\hline$\propto_{2}$ & $\frac{\pi}{2}$ & $\beta_{2}$ & 0 \\
\hline$\propto_{3}$ & $\pi-\tan ^{-1}\left(\frac{b}{2 c}\right)$ & $\beta_{3}$ & $\left(\frac{\pi}{2}+\gamma_{3}\right)-\propto_{3}$ \\
\hline$\propto_{4}$ & $-\pi+\tan ^{-1}\left(\frac{b}{2 c}\right)$ & $\beta_{4}$ & $\left(\frac{\pi}{2}+\gamma_{4}\right)-2 \pi-\propto_{4}$ \\
\hline$\propto_{5}$ & $-\frac{\pi}{2}$ & $\beta_{5}$ & $\pi$ \\
\hline$\propto_{6}$ & $-\tan ^{-1}\left(\frac{b}{2 a}\right)$ & $\beta_{6}$ & $\left(\frac{\pi}{2}+\gamma_{6}\right)-\propto_{6}$ \\
\hline
\end{tabular}

Where $\gamma_{i}$ represents the turning value of each wheel determined by calculating the instantaneous center of rotation.

As mentioned above, the mobile platform has the Rocker-Bogie system, which has directional wheels and two fixed wheels as shown Figure 5. Based on this, Figure 6 shows the configuration of the wheels, with their respective mathematical representation in equation Eq. (7) and Eq. (8). 

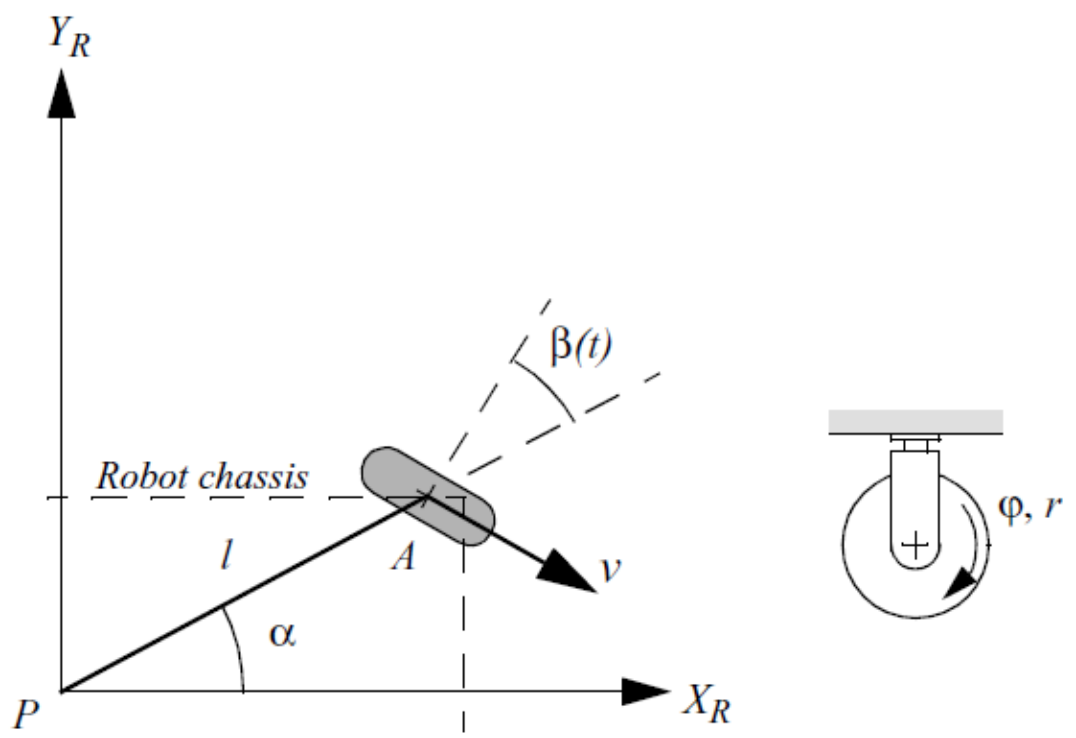

Fig. 6. Kinematic configuration of wheels

$$
\begin{gathered}
{[s(\alpha+\beta)-c(\alpha+\beta)-l \cdot c(\beta)] \cdot R(\theta) \cdot \dot{\xi}_{I}-r \dot{\varphi}=0} \\
{[c(\alpha+\beta) s(\alpha+\beta) l \cdot s(\beta)] \cdot R(\theta) \cdot \dot{\xi}_{I}-r \dot{\varphi}=0}
\end{gathered}
$$

Where $\dot{\xi}_{I}$ is composed of the global coordinates of the robot $[\dot{x}, \dot{y}, \dot{\theta}]^{T}, r$ is an identity matrix composed of the spokes of the wheels, and $R(\theta)$ is a rotational transformation matrix that describes the orientation of the robot in a global coordinate plane, as shown in Eq. (9).

$$
R(\theta)=\left[\begin{array}{ccc}
c(\theta) & s(\theta) & 0 \\
-s(\theta) & c(\theta) & 0 \\
0 & 0 & 1
\end{array}\right]
$$

Having the characteristic equations of the wheels, we proceed with the construction of the final kinematic representation that describes the movement of the mobile robotic platform, as shown in Eq. (10).

$$
\dot{\zeta}_{I}=R(\theta)^{-1}\left[\begin{array}{lll}
S_{\left(\alpha_{1}+\beta_{1}\right)} & -c_{\left(\alpha_{1}+\beta_{1}\right)} & -l_{1} \cdot c_{\left(\beta_{1}\right)} \\
s_{\left(\alpha_{2}+\beta_{2}\right)} & -c_{\left(\alpha_{2}+\beta_{2}\right)} & -l_{2} \cdot c_{\left(\beta_{2}\right)} \\
S_{\left(\alpha_{3}+\beta_{3}\right)} & -c_{\left(\alpha_{3}+\beta_{3}\right)} & -l_{3} \cdot c_{\left(\beta_{3}\right)} \\
S_{\left(\alpha_{4}+\beta_{4}\right)} & -c_{\left(\alpha_{4}+\beta_{4}\right)} & -l_{4} \cdot c_{\left(\beta_{4}\right)} \\
s_{\left(\alpha_{5}+\beta_{5}\right)} & -c_{\left(\alpha_{5}+\beta_{5}\right)} & -l_{5} \cdot c_{\left(\beta_{5}\right)} \\
S_{\left(\alpha_{6}+\beta_{6}\right)} & -c_{\left(\alpha_{6}+\beta_{6}\right)} & -l_{6} \cdot c_{\left(\beta_{6}\right)}
\end{array}\right]^{-1} \cdot\left[\begin{array}{cccccc}
r_{1} & 0 & 0 & 0 & 0 & 0 \\
0 & r_{2} & 0 & 0 & 0 & 0 \\
0 & 0 & r_{3} & 0 & 0 & 0 \\
0 & 0 & 0 & r_{4} & 0 & 0 \\
0 & 0 & 0 & 0 & r_{5} & 0 \\
0 & 0 & 0 & 0 & 0 & r_{6}
\end{array}\right] \cdot\left[\begin{array}{c}
\dot{\varphi_{1}} \\
\dot{\varphi_{2}} \\
\dot{\varphi_{3}} \\
\dot{\varphi_{4}} \\
\dot{\varphi_{5}} \\
\dot{\dot{\varphi}_{6}}
\end{array}\right]
$$

Where $l_{i}$ represent the distances from the center of mass of the dolly and each wheel and $\dot{\varphi}_{\imath}$ represent the speeds of each wheel. 


\section{$5 \quad$ Instant center of rotation.}

For calculating the instantaneous center of rotation, the geometry presented in Figure 7 is considered. From there, the equation of the circumference is proposed to calculate the turning radius that should be considered by the robot to go from a point $\left(x_{1}, y_{1}\right)$ located in the center of mass of the same, to a point $\left(x_{2}, y_{2}\right)$.

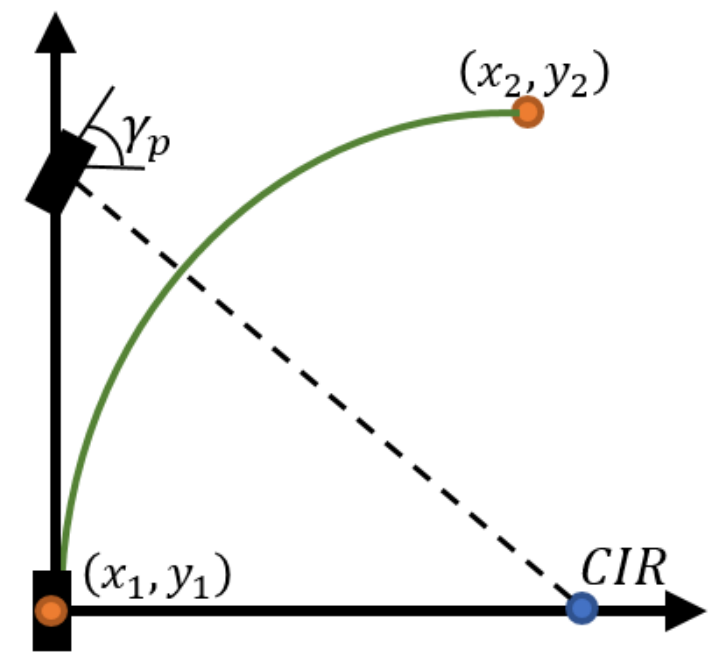

Fig. 7. Instant center of rotation (ICR).

To determine its instantaneous center of rotation, it is considered that $\left(x_{1}, y_{1}\right)$ and $\left(x_{2}, \mathrm{y}_{2}\right)$ are points of the circumference with center in $c(h, k)$ and that it is located on the line $\left(x_{1}, y_{1}\right),\left(r \cos (\theta)+x_{1} r \operatorname{sen}(\theta)+y_{1}\right)$, with slope $m=\operatorname{sen}(\theta) / \cos (\theta)$. With the above, the characteristic equations of the circle in Eq. (11) are posed, and since you have three equations and three unknowns, you proceed to solve the system of equations to obtain the values of $h, k, r$.

$$
\begin{gathered}
\left(x_{1}-h\right)^{2}+\left(y_{1}-k\right)^{2}=r^{2} \\
\left(x_{2}-h\right)^{2}+\left(y_{2}-k\right)^{2}=r^{2} \\
k-y_{1}-m\left(h-x_{1}\right)=0
\end{gathered}
$$

The calculation of $\gamma_{p}$ is performed by geometry taking into consideration Figure 8 and Eq. (12). To finally calculate the $\gamma_{i}$ described in Table 3.

$$
\begin{aligned}
& \gamma_{p}=\tan ^{-1}\left(\frac{a}{\left|h-x_{1}\right|}\right) \\
& \gamma_{m}=\tan ^{-1}\left(\frac{c}{\left|h-x_{1}\right|}\right)
\end{aligned}
$$




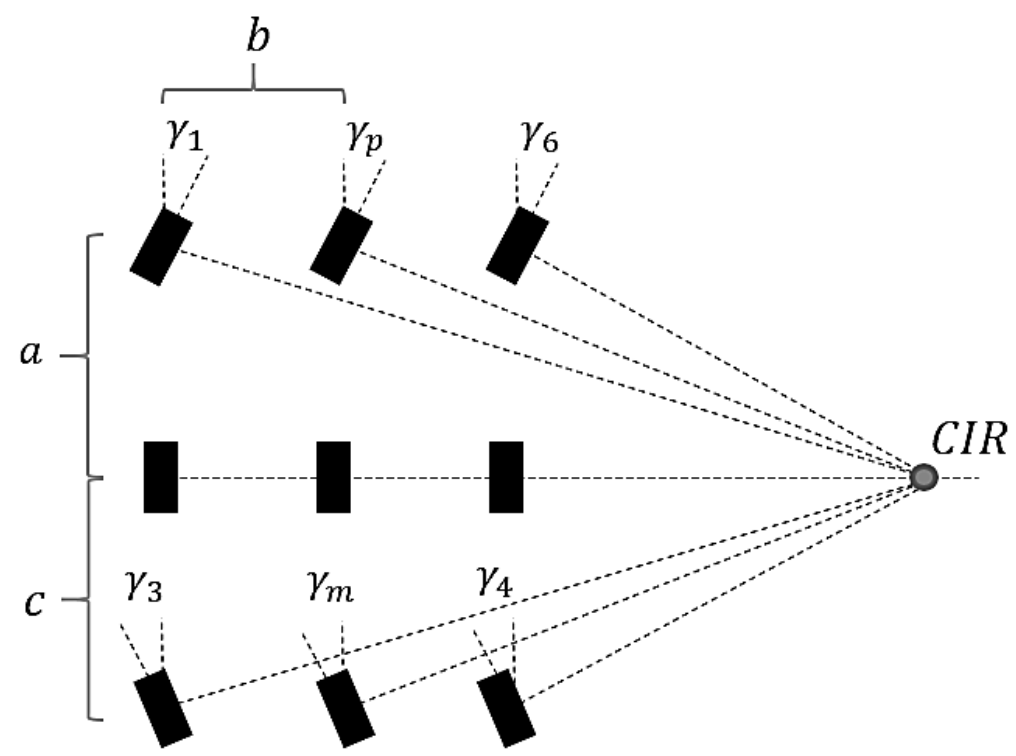

Fig. 8. Angular calculation of directional wheels.

\section{Simulation}

The robotic simulation software Webots will be used to verify the robotic system. It defines the properties of each object, its shape, color, texture, mass, friction, etc., as needed to emulate the robot in the virtual environment and generate physically realistic behaviors.

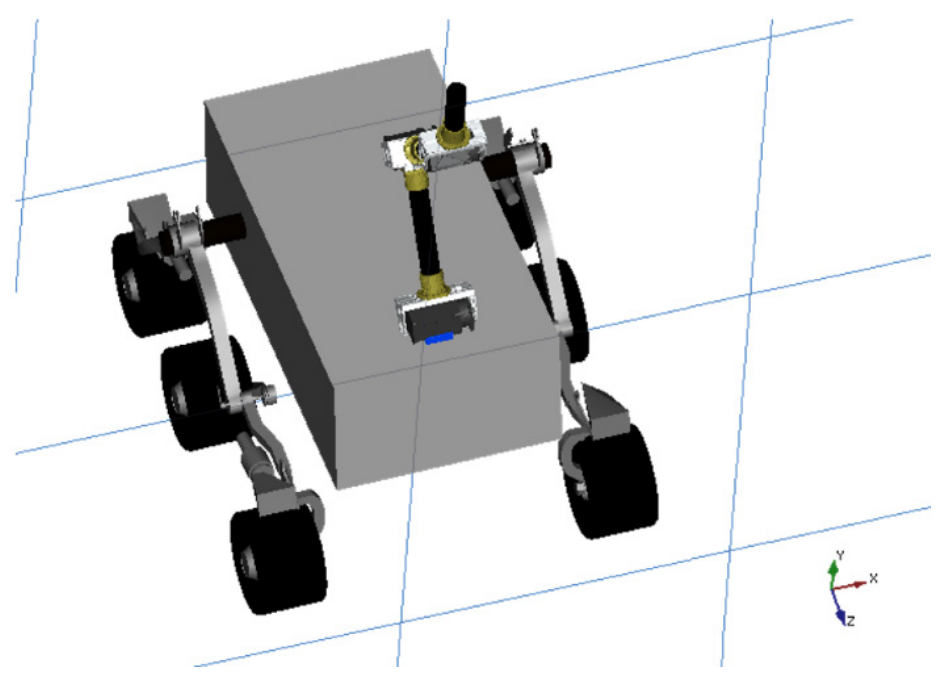

Fig. 9. Simplified robot $\mathrm{CAD}$ model. 
From a simplified CAD model of the assembled robot, it is imported into the virtual environment, which consists of a node tree that allows the hierarchy of WRML nodes that make up the virtual environment. In this node tree, in addition to defining the order of robot parts and how they relate to each other, physical properties such as weight, friction, mass centers, types of movements, collision meshes, etc. are defined.

In the virtual environment, in addition to the robot, a series of obstacles (stones and stairs) were added, as well as an irregular terrain that makes it possible to verify the robot's operation under these conditions and not only the correct operation of the designed programming. Webots allows to make use of controllers designed in MATLAB which allows from the mathematical models of the robot to design controllers and thus verify their behavior in the virtual environment. In addition to this by using a gamepad, the user is allowed to interact in the virtual environment by controlling the robot.

Figure 11 shows the robot passing through a terrain containing several rocks of different sizes, allowing verification of the Rocker-Bogie suspension in an irregular terrain. It is also evident that obstacles beyond the wheel diameter can be overcome, but there is a risk of robot tipping over.

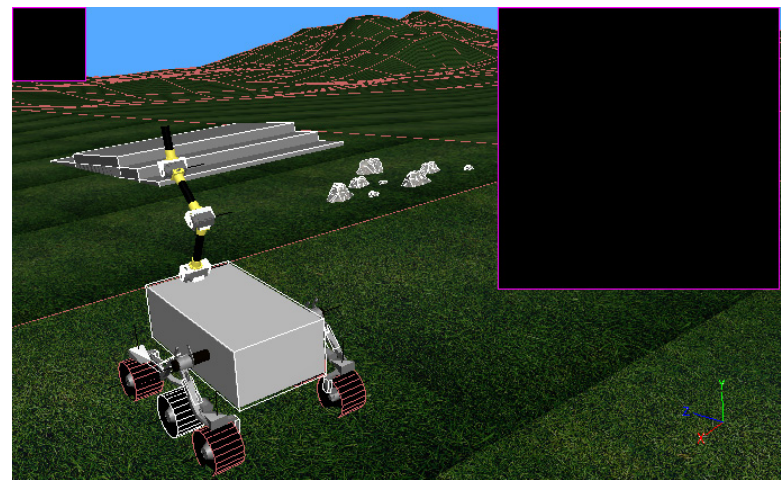

Fig. 10.Virtual Webots environment

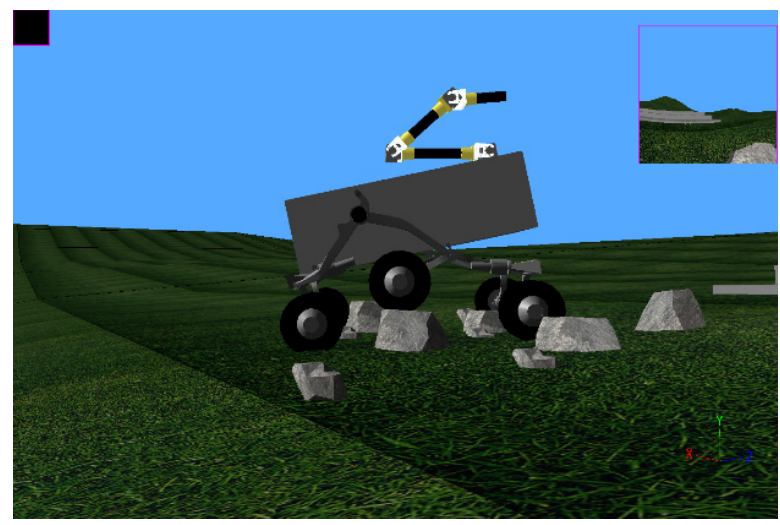

Fig. 11.Verification of suspension and traction. 


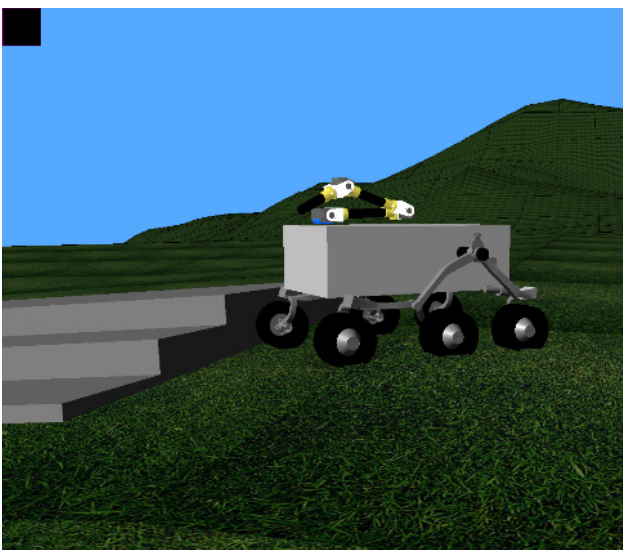

a) Initial movement of the manipulator.

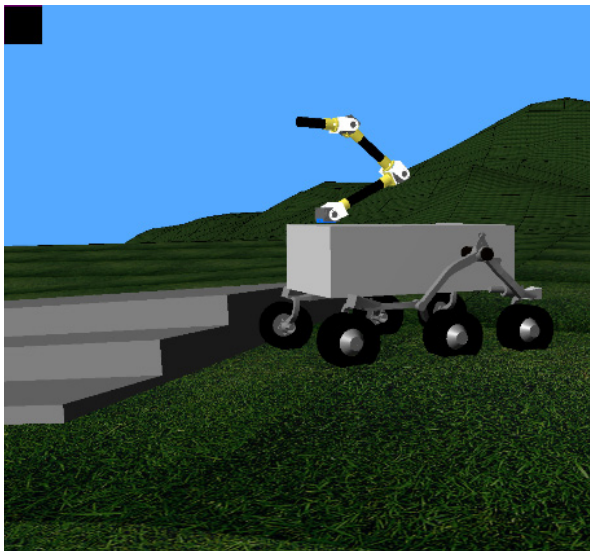

b) Final movement of the manipulator.

Fig. 12.Movement of the manipulator in the $\mathrm{Y}$ axis

As shown in Figure 12 the transition of the manipulator from an initial moment to a final moment on the $\mathrm{Y}$-axis is shown, verifying that the final effector moves in a straight line. To achieve this, the $\mathrm{P}$ vector found in the direct kinematics is used, proposing a system to be optimized. For this purpose, it was solved by means of the Newton - Rapshon successive approximations method, solving problems of infinite velocities or unwanted movements that could be generated by using the inverse kinematics in an analytical way from the position and orientation of the final effector, using equation (13).

$$
\left[\begin{array}{l}
\theta_{1}(i+1) \\
\theta_{2}(i+1) \\
\theta_{3}(i+1) \\
\theta_{4}(i+1)
\end{array}\right]=\left[\begin{array}{l}
\theta_{1}(i) \\
\theta_{2}(i) \\
\theta_{3}(i) \\
\theta_{4}(i)
\end{array}\right]-\frac{f\left(\theta_{1}(i), \theta_{2}(i), \theta_{3}(i), \theta_{4}(i)\right)}{f^{\prime \prime}\left(\theta_{1}(i), \theta_{2}(i), \theta_{3}(i), \theta_{4}(i)\right)}
$$

Where the cost function $\mathrm{f}(\mathrm{x})$ is defined as the position vector in $\mathrm{X}, \mathrm{Y}, \mathrm{Z}$ of the final link, which is obtained from the pre-multiplication of the transformation matrices.

$$
f\left(\theta_{1}(i), \theta_{2}(i), \theta_{3}(i), \theta_{4}(i)\right)=\left[\begin{array}{l}
\mathrm{X}-X_{d} \\
\mathrm{Y}-Y_{d} \\
\mathrm{Z}-Z_{d}
\end{array}\right]
$$

\section{Conclusions}

From a virtual environment, it is possible to simulate robots with complex characteristics in an environment that allow to compare their operation in complicated environments, in the same way as mathematical models and controllers designed for their operation. With this work, it was proved that the Rocker-Bogie system implemented for this project works correctly considering that its ability to overcome obstacles de- 
pends on the size of its wheels, also the speed with which it is intended to overcome obstacles as this could cause damage to the structure.

\section{Acknowledgements}

The research for this paper was supported by Nueva Granada Military University, through the project ING-IMP-2138

\section{$9 \quad$ References}

[1] P. M. Escobedo Castillo, «Manipulador móvil: estudio sobre la coordinación de movimientos de un manipulador serial acoplado», UNAM, Mexico, 2012.

[2] M. H. Korayem, V. Azimirad, A. Nikoobin, y Z. Boroujeni, «Maximum load-carrying capacity of autonomous mobile manipulator in an environment with obstacle considering tip over stability», Int. J. Adv. Manuf. Technol., vol. 46, n.o 5-8, pp. 811-829, ene. 2010.

[3] «Springer Handbook of Robotics | Bruno Siciliano | Springer». [En línea]. Disponible en: http://www.springer.com/us/book/9783540382195. [Accedido: 17-nov-2016].

[4] J. B. Cano, M. A. Giraldo, D. L. Mazo, N. L. Ospina, y N. D. M. C, «Sistema de control y comando de un robot móvil para terrenos irregulares», Rev. POLITÉCNICA, vol. 4, n.o 6, pp. 39-52, feb. 2015.

[5] Miller David P. y Lee Tze-Liang, «High-Speed Traversal of Rough Terrain Using a Rocker-Bogie Mobility System», Space 2002 Robot. 2002.

[6] M. Thianwiboon y V. Sangveraphunsiri, «Traction Control for a Rocker-Bogie Robot with Wheel-Ground Contact Angle Estimation», en RoboCup 2005: Robot Soccer World Cup IX, 2005, pp. 682-690.

[7] L. Apostolovich y L. Felipe, «Modelación y simulación dinámica de un brazo robótico de 4 grados de libertad para tareas sobre un plano horizontal», Pontif. Univ. Católica Perú, may 2011.

\section{Authors}

Óscar F. Avilés S Was born in Bogotá, Colombia, in 1967. He received the Engineer degree in Electronics and Specialist of Electronic Instrumentation of Antonio Nariño University - UAN - in 1995 and 2002, respectively. Master in Automatic Production Systems - Technological University of Pereira - 2006 Colombia and PhD in Mechanical Engineering in Campinas State University - UNICAMP - Brazil. Currently is Professor in the Department of Mechatronics Engineering in Militar Nueva Granada University - UMNG. He has experience in the areas of Electronic Engineering, with emphasis on electronic instrumentation and control systems, acting mainly on the following topics: robotics, control and biomechatronics.

Oscar G. Rubiano M was born in Colombia in 1990. He studied his undergraduate in Militar Nueva Granada University where he received a degree in Mechatronics Engineering in 2013. He is a student of Mechatronics Engineering Master in Nueva 
Granada University. He has experience in the areas of modular robotics, mobile autonomous robotics and collaborative robotics.

Mauricio F. Mauledoux M was born in Bogotá, Colombia, in 1982. He received the Engineer degree in Mechatronic engineering from the Militar Nueva Granada University, in 2005. In 2008 as a student of the Master in Information Technologies and Intelligent Systems in the St. Petersburg State Polytechnic University, Russia, at the automatic and intelligent distributed control department, he was promoted to a $\mathrm{PhD}$. In $2011 \mathrm{He}$ received the $\mathrm{PhD}$ degree in Mathematical models, numerical methods and software systems (Red Diploma) from the St. Petersburg State Polytechnic University, Russia. In 2012, he joined the Department of Mechatronic Engineering, at Militar Nueva Granada University, in Colombia, as an Assistant Professor. His current research interests include Robotics, automatic control, Multi-agent Systems, Smart Grids, and Optimization.

Angie J. Valencia C. was born in Bogotá, Colombia, in 1994. She received the B.S degree in Mechatronics engineering from the University Militar Nueva Granada, Colombia, in 2015. In this year, she joined the Davinci Group at the Militar Nueva Granada University, Colombia, as researching assistant. Her current research interests include Control System, Quadrotor prototype, Robotics, and Optimization.

Robinson Jiménez Moreno was born in Bogotá, Colombia, in 1978. He received the Engineer degree in Electronics from the Francisco José de Caldas district University - UD - in 2002, respectively. Master in Industrial Automation from the Universidad Nacional de Colombia - 2012 and third year PhD student in Engineering of the Francisco José de Caldas district University - UD. He is currently an Assistant Professor in the Mechatronics Engineering Program of the Nueva Granada Military University - UMNG. He has experience in the areas of Instrumentation and Electronic Control, acting mainly in: Robotics, Control, pattern recognition and image processing.

Article submitted 02 October 2017. Final acceptance 08 December 2017. Final version published as submitted by the authors. 